\title{
ETHNOZOOLOGIE DE LA FAUNE MAMMALOGIQUE DE LA FORET CLASSEE DE BADENOU (KORHOGO, CÔTE-D'IVOIRE)
}

\author{
H. K. YAOKOKORE-BEIBRO, B. K. KASSE, O. SOULEMANE, M. T. KOUE-BI, P. K. KOUASSI et K. FOUA-BI \\ Laboratoire de Zoologie et Biologie Animale, UFR Biosciences, Université de Cocody, Abidjan, 22 BP 582 Abidjan 22. \\ E-mail : hyaokokore@yahoo.fr
}

\begin{abstract}
RESUME
La faune des mammifères de la forêt classée de Badénou (Korhogo) est peu connue. Une étude ethnozoologique et des inventaires fauniques entre 1994 et 1998 ont permis de dresser, pour la première fois, une liste de 45 espèces de mammifères appartenant à 36 genres, 20 familles et 9 ordres. Les résultats montrent une faune bien connue des populations riveraines. Cette faune joue un rôle important, dans l'alimentation et les us et coutumes. L'utilisation des produits secondaires de la faune, et de la chasse de subsistance, demeurent incontrôlés et s'orientent vers la seule demande de marché. Elle correspond ainsi à un système d'exploitation indépendant du revenu mais lié au capital forestier.
\end{abstract}

Mots clés : Diversité des mammifères, ethnozoologie, connaissances locales, Côte d'Ivoire.

\author{
ABSTRACT \\ ETHNOZOOLOGY OFBADENOU NATIONAL FOREST (KORHOGO, CÔTED'IVOIRE)
}

The Mammals fauna of the Badénou National Forest in Korhogo, north of Côte d'Ivoire, is poorly known. An ethnozoological survey was conducted from 1994 to 1998, which revealed a total 45 species of 36 geniuses, belonging to 20 families from 9 orders recorded for the first time in this forest. The results revealed that the people living in the outskirts of the Badénou National Forest have a very good knowledge of the fauna. This fauna contributed significantly in their feeding habit as a major source of animal protein, in the local customs and practice. The use of the secondary products of the fauna, including subsistence hunting is not under control.

Key words : Mammals' diversity, ethnozoology, local knowledge, Côte d'Ivoire.

\section{INTRODUCTION}

Les milieux naturels africains constituent, pour les populations locales, un domaine vital aux plans écologique, économique et social. Ils représentent d'importants réservoirs de biodiversité. Leur disparition s'est accentuée ces dernières décennies au point de menacer les civilisations, aux populations peu nombreuses, qui en sont largement tributaires (Daniel, 1992).

Parmi les ressources potentielles de ces milieux naturels, la faune tient une place de choix (Wolf, 1991 ; Brousseau, 1992 a et b). Pour mener des actions de sauvegarde efficaces, il est nécessaire d'associer les populations locales dans leur politique de gestion de ces espaces
(Haldik, 1992). En effet, les populations apprécient les ressources biologiques de manières différentes : spirituellement, économiquement, esthétiquement, culturellement et scientifiquement (Anonyme 1, 1993). Les comportements des individus et des groupes, leurs actions, influencent leur environnement et assurent le contact entre les systèmes écologiques et sociaux. Les comportements fournissent donc une fenêtre sur ces systèmes, afin de mieux comprendre le contexte et les motivations intrinsèques à la consommation et à la gestion des ressources naturelles et afin d'influencer ces comportements et de promouvoir la durabilité (Byers, 1997 ; Caspary et al., 2001). C'est pourquoi, il est nécessaire de connaître les us et coutumes des 
populations locales, afin d'en tenir compte dans la gestion de nos forêts. Cette ethnozoologie, science des différents usages des animaux de la faune spécifique par une ou plusieurs communautés ethniques, occupe une place de choix dans le processus de la gestion durable de la forêt classée de Badénou (FCB) à Korhogo (Yaokokoré-Béibro, 1995).

\section{MATERIEL ET METHODES}

\section{SITE D'ETUDE}

La forêt classée de Badénou couvre une superficie 26980 ha et est située au Nord de la Côte d'Ivoire ( $5^{\circ} 32^{\prime} 06^{\prime \prime}$ et $5^{\circ} 49^{\prime} 67^{\prime \prime} \mathrm{W} ; 9^{\circ} 41^{\prime}$ $63^{\prime \prime}$ et $\left.9^{\circ} 51^{\prime} 63^{\prime \prime} \mathrm{N}\right)$, à $40 \mathrm{~km}$ de la ville de Korhogo et à $23 \mathrm{~km}$ au sud-est de M'bengué (Figure 1). Elle est caractérisée à un climat tropical de type soudano-guinéen à deux saisons (une saison sèche de novembre à avril, et une saison de pluie qui s'étend de mai à octobre. Elle connaît un maximum en août et en septembre). L'essentiel des précipitations se concentre sur la période de juillet à septembre avec des pluviométries moyennes annuelles autour de $1200 \mathrm{~mm}$. Les températures moyennes annuelles sont voisines de $27^{\circ} \mathrm{C}$ et oscille entre $29^{\circ} \mathrm{C}$ entre février - mars et $24^{\circ} \mathrm{C}$ en août (Anonyme 2, 1992 ; Yaokokoré-Béibro, 1995 ; Kassé et al., 2006). Elle appartient à la zone phytogéographique sub-soudanienne avec une mosaïque de forêts et de savanes où l'on trouve des îlots de forêts denses sèches, des forêts claires, des forêts galeries, des savanes arborées, des savanes arbustives, et des savanes herbeuses.

La population riveraine de la FCB est composée essentiellement, de Sénoufos et de Malinkés (ethnies autochtones) généralement animistes ou musulmans. A ces autochtones s'ajoutent des allochtones (Attié, Baoulé, Bété, Lobi) et des allogènes venus des pays voisins (Mali, Burkina Faso, Guinée). Cette population est estimée à 1000 personnes, dont 500 vivent en permanence à l'ntérieur de la forêt. Elle est dans sa grande majorité paysanne (YaokokoréBéibro, 1995).

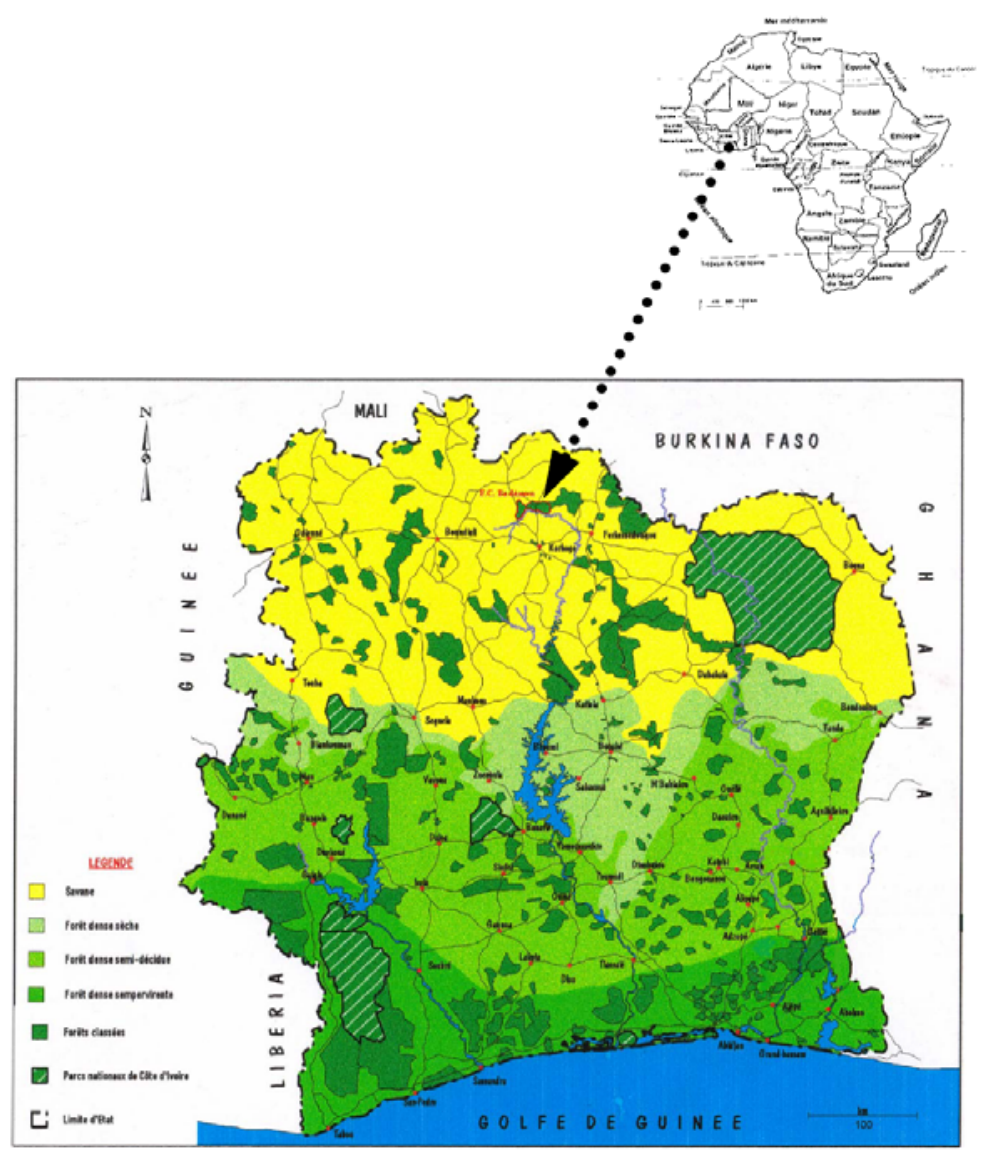

Figure 1 : Localisation de la forêt classée de Badénou dans le Département de Korhogo..

Badenou National Forest localisation in the Korhogo Department. 


\section{COLLECTE DES DONNEES}

La collecte des données a été réalisée à partir de fiches d'enquêtes et des entretiens. Les entretiens ont été réalisés avec les populations. Les interviews, participatives, ont été réalisés de novembre 1994 à novembre 1998 (Gouet et al., 1978).

L'étude a été menée dans 9 des 10 villages et campements, situés à l'intérieur et à la périphérie de la FCB. Seuls, les hommes ont été interrogés, car, les plus directement impliqués dans les activités en relation avec la faune sauvage. Elle a été menée avec la collaboration de personnes issues de différentes catégories sociales ayant une bonne connaissance de la faune et de ses différents usages (chefs de village, notables, chasseurs, paysans et jeunes hommes). L'interview a été identique pour toutes ces catégories sociales. Les réunions communautaires ont été planifiées et animées par les enquêteurs, pour minimiser les antagonismes au sein de la communauté (Byers, 1977). Des fiches d'enquête et un dictaphone ont été utilisés comme support d'enquête et pour l'enregistrement des conversations. Les services d'un interprète local ont été sollicités pour la traduction des entretiens en langue Sénoufo et Malinké. La présence d'un ancien braconnier reconverti a facilité la communication avec les différents groupes cibles.

Les visites régulières de terrain, à raison de deux par semaine pendant toute la durée de l'étude, le long des layons à l'intérieur de la FCB, ont permis de compléter les données d'enquête (Kassé et al., 2006) et de confirmer la présence des espèces. L'identification des espèces de mammifères a été faite à partir du guide des mammifères d'Afrique et de Madagascar (Haltenorth et al., 1985). Dans cette étude, les espèces sont dites " peu communes " Iorsqu'au moins un individu a été observé au cours de l'année. Elles sont dites " communes " lorsque ce nombre varie de un à neuf dans l'habitat adéquat à chaque visite de terrain. Elles sont dites " abondantes " Iorsque ce nombre est supérieur ou égal à 10 dans un habitat adéquat.

Les travaux qui ont porté sur les grands et moyens mammifères ont permis de dresser une liste des espèces présentes dans la FCB, d'obtenir les indices de présence, les statuts, d'en connaître les noms en langues locales (Sénoufo et Malinké), le mode de capture, ainsi que les différents usages par les populations riveraines.

\section{RESULTATS}

L'étude a été menée avec la collaboration de 86 individus représentant un taux de sondage de $17,55 \%$.

\section{RICHESSE DE LAFAUNE MAMMALIENNE}

La faune mammalienne de la FCB est riche de 45 espèces reparties en 36 genres de 20 familles et de 9 ordres (Tableau 1). Parmi les espèces présentes, 7 ont eu une présence incertaine (douteuse) ; Dix sept sont peu communes, 10 sont communes et seulement 7 peuvent être considérées comme abondantes.

\section{CONNAISSANCE LOCALE DE LAFAUNE}

La quasi-totalité des espèces de mammifères possèdent un nom sénoufo et malinké, montrant ainsi la bonne connaissance des populations locales de leur faune. Au moins 20 espèces ont été reconnues sous au moins 2 noms senoufo distincts, contre 9 espèces en malinké (Tableau 2). Dans tous les ordres présents, seuls les Carnivores ont eu des espèces qui portent le même nom local. Six espèces portent par binôme générique les mêmes noms sénoufos, ce qui laisse supposer que le Sénoufo les reconnaît et peut décrire les espèces concernées jusqu'au niveau taxonomique du genre. Seul le babouin (Papio doguera) a le même nom en sénoufo et en malinké. Pour toutes les autres espèces, chaque groupe ethnique possède un nom qui lui est propre. 
Tableau 1 : Principales espèces de mammifères de la forêt classée de Badénou et leurs indices de présence.

Main mammals species of Badenou National Forest and their presence indice.

\begin{tabular}{|c|c|c|}
\hline $\begin{array}{l}\text { Nom scientifique } \\
\text { Ordre/famille/sous-famille/espèce }\end{array}$ & Nom français & Indice de présence \\
\hline Pholidota & Pholidotes & \\
\hline \multicolumn{3}{|l|}{ Manidae } \\
\hline Manis gigantea Illiger, 1815 & Pangolin géant & (x) \\
\hline Insectivora & Insectivores & \\
\hline \multicolumn{3}{|l|}{ Erinaceidae } \\
\hline Atelerix albiventris (Wagner, 1841) & Hérisson à ventre blanc & $\mathrm{xxx}$ \\
\hline Carnivora & Carnivores & \\
\hline \multicolumn{3}{|l|}{ Canidae } \\
\hline Canis adustus Sundevall, 1847 & Chacal à flancs rayés & $\mathrm{x}$ \\
\hline Lycaon pictus (Temminck, 1820) & Lycaon & (x) \\
\hline \multicolumn{3}{|l|}{ Mustelidae } \\
\hline Mellivora capensis (Schreber, 1776) & Ratel & (x) \\
\hline \multicolumn{3}{|l|}{ Viverridae } \\
\hline Genetta thierryi Matschie, 1902 & Genette de Villiers & $\mathrm{xx}$ \\
\hline Genetta tigrina (Schreber, 1776) & Genette tigrine & $\mathrm{xx}$ \\
\hline Civettictis civetta (Schreber, 1776) & Civette & $\mathrm{x}$ \\
\hline \multicolumn{3}{|l|}{ Herpestidae } \\
\hline Herpestes sanguineus Rüppel & Mangouste rouge & $\mathrm{xx}$ \\
\hline Atilax paludinosus (G. [Baron] Cuvier, 1829) & Mangouste des marais & $\mathrm{x}$ \\
\hline Herpestes ichneumon (Linné, 1758) & Mangouste ichneumon & (x) \\
\hline Crossarchus obscurus F. G. Cuvier, 1825 & Mangouste brune & $\mathrm{x}$ \\
\hline Ichneumia albicauda (G. [Baron] Cuvier, 1829) & Mangouste à queue blanche & $\mathrm{x}$ \\
\hline Mungos gambianus (Ogilby, 1835) & Mangue de Gambie & $\mathrm{x}$ \\
\hline \multicolumn{3}{|l|}{ Hyaenidae } \\
\hline Crocuta crocuta (erxleben, 1777) & Hyène tachetée & \\
\hline \multicolumn{3}{|l|}{ Felidae } \\
\hline Panthera leo (Linné, 1758) & Lion & $(\mathrm{x})$ \\
\hline Panthera pardus (Linné, 1758) & Panthère & $(\mathrm{x})$ \\
\hline Caracal caracal (Schreber, 1776) & Caracal & $\mathrm{x}$ \\
\hline Primates & Primates & \\
\hline \multicolumn{3}{|l|}{ Galagonidae } \\
\hline Galago senegalensis É. Geoffroy St-Hilaire, 1796 & Galago du Sénégal & $\mathrm{xx}$ \\
\hline \multicolumn{3}{|l|}{ Cercopithecidae } \\
\hline Papio anubis doguera & Babouin & $\mathrm{x}$ \\
\hline Erythrocebus patas (Schreber, 1775) & Singe rouge & $\mathrm{xxx}$ \\
\hline Cercopithecus aethiops (Linné, 1766) & Vervet & $\mathrm{x}$ \\
\hline Lagomorpha & Lagomorphes & \\
\hline \multicolumn{3}{|l|}{ Leporidae } \\
\hline Lepus saxatilis F. Cuvier, 1823 & Lièvre & $\mathrm{xxx}$ \\
\hline Rodentia & Rongeurs & \\
\hline \multicolumn{3}{|l|}{ Hystricidae } \\
\hline Hystrix cristata Linné, 1758 & Porc-épic & $\mathrm{xxx}$ \\
\hline \multicolumn{3}{|l|}{ Muridae } \\
\hline Cricetomys gambianus Waterhouse, 1840 & Rat de Gambie & $\mathrm{xxx}$ \\
\hline
\end{tabular}


(Suite)

\begin{tabular}{|c|c|c|}
\hline \multicolumn{3}{|l|}{ Thryonomyidae } \\
\hline Thryonomys swinderianus (Temminck, 1827) & Grand aulacode & $\mathrm{xx}$ \\
\hline \multicolumn{3}{|l|}{ Sciuridae } \\
\hline Xerus erythropus (Desmarest, 1817) & Écureuil fouisseur & $\mathrm{xxx}$ \\
\hline Funisciurus pyrropus (F. Cuvier, 1833) & Funisciure à pattes rousses & $\mathrm{xxx}$ \\
\hline \multicolumn{3}{|l|}{ Anomaluridae } \\
\hline Anomalurus pelii (Schlegel \& Müller, 1845) & Écureuil volant & $\mathrm{x}$ \\
\hline Tubulidentata & Tubulidentés & \\
\hline \multicolumn{3}{|l|}{ Orycteropidae } \\
\hline Orycteropus afer (Pallas, 1766) & Oryctérope & $\mathrm{x}$ \\
\hline Artiodactyla & Artiodactyles & \\
\hline \multicolumn{3}{|l|}{ Suidae } \\
\hline Phacochoerus aethiopicus (Pallas, 1766) & Phacochère & $\mathrm{xx}$ \\
\hline \multicolumn{3}{|l|}{ Hippopotamidae } \\
\hline Hippopotamus amphibius Linné, 1758 & Hippopotame & $\mathrm{x}$ \\
\hline \multicolumn{3}{|l|}{ Bovidae } \\
\hline \multicolumn{3}{|l|}{ Cephalophinae } \\
\hline Cephalophus maxwellii (H. Smith, 1827) & Céphalophe de Maxwell & (x) \\
\hline Cephalophus niger Gray, 1846 & Céphalophe noir & $\mathrm{xx}$ \\
\hline Cephalophus silvicultor (Afzelius, 1815) & Céphalophe à dos jaune & $(\mathrm{x})$ \\
\hline Cephalophus rufilatus Gray, 1846 & Céphalophe à flancs roux & $\mathrm{xx}$ \\
\hline Cephalophus dorsalis Gray, 1846 & Céphalophe à bande dorsale noire & \\
\hline Ourebia ourebi (Zimmermann, 1783) & Ourébi & $\mathrm{x}$ \\
\hline \multicolumn{3}{|l|}{ Tragelaphinae } \\
\hline Tragelaphus scriptus (Pallas, 1766) & Guib harnaché & $\mathrm{xx}$ \\
\hline \multicolumn{3}{|l|}{ Reduncinae } \\
\hline Kobus ellipsiprymnus defassa (Ogilby, 1833) & Cobe defassa & $(\mathrm{x})$ \\
\hline Kobus kob (Erxleben, 1777) & Cobe de Buffon & $\mathrm{xx}$ \\
\hline Redunca redunca (Pallas, 1767) & Nagor & $\mathrm{x}$ \\
\hline Hippotragus equinus (Desmarest, 1804) & Antilope rouanne & $\mathrm{x}$ \\
\hline \multicolumn{3}{|l|}{ Alcelaphinae } \\
\hline Alcelaphus buselaphus (Pallas, 1766) & Bubale & $\mathrm{x}$ \\
\hline \multicolumn{3}{|l|}{ Bovinae } \\
\hline Syncerus caffer (Sparrman, 1779) & Buffle & $\mathrm{x}$ \\
\hline
\end{tabular}

$(x)$ : présence douteuse ; $x$ : espèce peu commune ; $x x$ : espèce commune ; $x x x$ : espèce abondante ;

spéci:. spécimen 
Tableau 2 : Noms en langues locales des mammifères connus par les populations riveraines de la forêt classée de Badénou.

Local name of mammals used by the local people in the Badenou National Forest.

\begin{tabular}{|c|c|c|c|}
\hline Nom scientifique & Nom français & Nom sénoufo & Nom malinké \\
\hline Pholidota & Pholidotes & & \\
\hline Manis giganea & Pangolin géant & Kromandague/Korodingue & Pkorokara \\
\hline Insectivora & Insectivores & & \\
\hline Erinaceus albiventris & Hérisson à ventre blanc & Térémitchole/Crintcholo/Didinnan/Dandaga & Djoukouni/Gnoukouni \\
\hline Carnivora & Carnivores & & \\
\hline Canis adustus & Chacal à flancs rayés & Solomanca/Soleca/Tainnibonnon/Gbague/Cépon & Kogowourou \\
\hline Lycaon pictus & Lycaon & Solomicargue & Kogowourou \\
\hline Mellivora capensis & Ratel & Sandrontin & \\
\hline Genetta thierryi & Genette de Villiers & Gonnon & Djakouman-woura \\
\hline Genetta tigrina & Genette tigrine & Gonnon & Djakouman-woura \\
\hline Viverra civetta & Civette & Tarague/Tâlégué/Temelon & Bakôrônigourou \\
\hline Herpestes sanguineus & Mangouste rouge & Tannigbannan/Tiganizinde/Dangbanne & Winzinni/Sissaimlan woura \\
\hline Herpestes paludinosus & Mangouste des marais & Tannangan/Srôgue/Djôgue & Winzinni/Sissaimlan \\
\hline Herpestes ichneumon & Mangouste ichneumon & Tannangan/Srôgue/Djôgue & Winzinni/Sissaimlan \\
\hline Mungos obscurus & Mangouste brune & Fohowélé & Winzinni/Sissaimlan \\
\hline Mungos gambianus & Mangue de Gambie & Fohowélé & Winzinni/Sissaimlan \\
\hline Ichneumia albicauda & Mangouste à queue blanche & Djônanvigue & Winzinni/Sissaimlan/woura \\
\hline Crocuta crocuta & Hyène tachetée & Kadall & Srougou/Souroukou \\
\hline Panthera leo & Lion & Djara/Tchara & Wara \\
\hline Panthera pardus & Panthère & Cédéo & Waranikara \\
\hline Caracal caracal & Caracal & Tougnimbon/Tainnebon/Tougnombon & \\
\hline Primates & Primates & & \\
\hline Galago senegalensis & Galago du Sénégal & Nannarga/Narga/Nannargahô & Bêtoloni \\
\hline Papio doguera & Babouin & Gbon & Gbon \\
\hline Erythrocebus patas & Singe rouge & Kôhtounnon/Kôhtounn yinhon & Soura woulé \\
\hline Cercopithecus aethiops & Vervet ou Singe vert & Kôhlo & Kogognon \\
\hline Lagomorpha & Lagomorphes & & \\
\hline Lepus saxatilis & Lièvre & Piéll/béng & Sozanni \\
\hline Rodentia & Rongeurs & & \\
\hline Hystrix cristata & Porc-épic & Yiriou & Balla \\
\hline Cricetomys gambianus & Rat de Gambie & Ganan/Gana & Toto \\
\hline $\begin{array}{l}\text { Thryonomys } \\
\text { swinderianus }\end{array}$ & Grand Aulacode & Wounou/Mounou & Kogninan \\
\hline Xerus erythropus & Écureuil fouisseur & Darsong/Dessong & N'gèlèni \\
\hline Funisciurus pyrrhopus & Funisciure à pattes rousses & Lossanni/Baitôllo/Tôllo & Wiri-tâ n'gèlèni \\
\hline Anomalurus sp & Écureuil volant & Lopié/Kolfargua & Cklontè-waratè \\
\hline Tubulidentata & Tubulidentés & & \\
\hline Orycteropus afer & Oryctérope & Gnarahô & Toumba/Timeba \\
\hline Artiodactyla & Artiodactyles & & \\
\hline $\begin{array}{l}\text { Phacochoerus } \\
\text { aethiopicus }\end{array}$ & Phacochère & Tchalahô/Tiahou & Lai \\
\hline $\begin{array}{l}\text { Hippopotamus } \\
\text { amphibius }\end{array}$ & Hippopotame & Koundro & Méri/Méli \\
\hline Cephalophus maxwelli & Céphalophe de Maxwell & Sôfilè & Goloni \\
\hline Cephalophus niger & Céphalophe noir & Sôwollô/Silaile/Silailè/Kpallailè & Kôkoulani fiman \\
\hline Cephalophus sylvicultor & Céphalophe à dos jaune & Dangoumon/Songrommon/Dangbon & Kômissi \\
\hline Cephalophus rufilatus & Céphalophe à flancs roux & Sôtchonn & Kôkoulani wouléman \\
\hline Ourebia ourebi & Ourébi & Sôfonnon & \\
\hline Tragelaphus scriptus & Guib harnaché & Kaffahô/Kaffa/Tchêng & M'nan/Minan \\
\hline Kobus defassa & Cobe defassa & Jahô yinhon & Sézé wouléman \\
\hline Kobus kob & Cobe de Buffon & Wollô/Oga/Wéléo & Son \\
\hline Redunca redunca & Nagor & Ognarahô/Ogonnon/yinhon & Kongoro wouléman \\
\hline Hippotragus equinus & Antilope rouanne & Gnon yinhon & Dagbè wouléman \\
\hline Alcelaphus buselaphus & Bubale & Yôh & Tangô \\
\hline Syncerus caffer & Buffle & Sénon & Kogomissi/Sigui \\
\hline
\end{tabular}




\section{UTILISATION DE LAFAUNE MAMMALIENNE}

La faune de la FCB est une source de protéine animale constituant un aliment essentiel pour les populations. Elle intervient également dans les us et coutumes, dans la médecine traditionnelle et les activités commerciales (Tableau 3).

Tableau 3 : Quelques utilisations des Mammifères de la forêt classée de Badénou. Some mammals' uses of local people in the Badenou national forest.

\begin{tabular}{|c|c|c|c|}
\hline Taxons & Méthode de prélèvement & Pharmacopée & Culturels \\
\hline Babouin & $\begin{array}{l}\text { Chasse au fusil, avec ou sans } \\
\text { chien. }\end{array}$ & $\begin{array}{l}\text { La paume est utilisée dans le } \\
\text { traitement des piqûres de } \\
\text { scorpions. }\end{array}$ & \\
\hline Bubale & Chasse au fusil & & $\begin{array}{l}\text { Ses cornes servent à fabriquer des trompettes; } \\
\text { la peau sert à fabriquer des tam-tams; les } \\
\text { tendons servent de substrats de poudre } \\
\text { d'allumette pour les fusils artisanaux. }\end{array}$ \\
\hline Céphalophes & $\begin{array}{l}\text { Chasse au fusil avec ou sans } \\
\text { l'aide des chiens ; parfois } \\
\text { capturés avec des pièges } \\
\text { artisanaux. }\end{array}$ & $\begin{array}{l}\text { La consommation de la chair } \\
\text { est un remède contre les } \\
\text { anémies } \\
\text { Les cornes sont utilisées pour } \\
\text { conserver divers } \\
\text { médicaments et fétiches. }\end{array}$ & $\begin{array}{l}\text { La peau sert à fabriquer de fouets et des } \\
\text { instruments de musique: coras, balafons, tam- } \\
\text { tams. Les tendons servent à attacher le canon et } \\
\text { la crosse des fusils artisanaux, et sont utilisés } \\
\text { comme substrat de poudre d'allumette servant } \\
\text { au déclenchement des fusils. }\end{array}$ \\
\hline Cobes & Chasse au fusil & $\begin{array}{l}\text { Les cornes servent à } \\
\text { conserver divers } \\
\text { médicaments et fétiches. }\end{array}$ & $\begin{array}{l}\text { La peau sert de couchette et à fabriquer des } \\
\text { tam-tams et des balafons; les tendons servent } \\
\text { de corde pour le canon et la crosse des fusils } \\
\text { artisanaux. La queue sert de trophée. }\end{array}$ \\
\hline Écureuils & $\begin{array}{l}\text { Chasse par piégeage avec l'aide } \\
\text { de chiens, de lance-pierres.et de } \\
\text { gourdins. }\end{array}$ & $\begin{array}{l}\text { La peau réduite en cendre et } \\
\text { mélangée avec du beurre de } \\
\text { karité est un remède contre } \\
\text { les maux d'yeux. }\end{array}$ & chassé et consommé par les enfants. \\
\hline Aulacodes & $\begin{array}{l}\text { Chasse au fusil, avec ou sans } \\
\text { chien et parfois par piégeage. }\end{array}$ & $\begin{array}{l}\text { les poils réduits en cendre } \\
\text { sont utilisés comme remède } \\
\text { contre les piqûres d'insectes } \\
\text { et d'épines d'arbre. }\end{array}$ & \\
\hline Guibs & $\begin{array}{l}\text { Chasse au fusil, avec ou sans } \\
\text { chien, rarement avec des pièges. }\end{array}$ & $\begin{array}{l}\text { les cornes sont utilisées pour } \\
\text { conserver les médicaments } \\
\text { contre les lombalgies et les } \\
\text { morsures de serpent. }\end{array}$ & $\begin{array}{l}\text { La peau sert de couchette et de tapis de prière; } \\
\text { les tendons servent de substrat de poudre } \\
\text { d'allumette pour les fusils artisanaux et de } \\
\text { corde pour attacher le canon et la crosse de ces } \\
\text { fusils. Il est un totem pour les charlatans ("san- } \\
\text { dolé"). }\end{array}$ \\
\hline Hippopotames & Chasse au fusil & $\begin{array}{l}\text { La chair est un remède contre } \\
\text { le rhumatisme articulaire; La } \\
\text { graisse est un anti- poisons; }\end{array}$ & $\begin{array}{l}\text { Animal maléfique qui peut entraîner le déclin } \\
\text { du village où vit le chasseur qui l'abat } \\
\text { Le chasseur peut devenir stérile s'il n'est pas } \\
\text { bien "purifié". } \\
\text { La queue doit être coupée et jetée dans l'eau } \\
\text { avant de transporter la chair au village. }\end{array}$ \\
\hline Hippotragues & Chasse au fusil & $\begin{array}{l}\text { Les cornes servent à } \\
\text { conserver divers fétiches. }\end{array}$ & $\begin{array}{l}\text { La peau sert de tapis. La queue est un trophée } \\
\text { pour le chasseur. }\end{array}$ \\
\hline Taxons & Méthode de prélèvement & Pharmacopée & Culturels \\
\hline Lièvres & $\begin{array}{l}\text { Chasse au fusil avec ou sans } \\
\text { chien, rarement par piégeage. }\end{array}$ & $\begin{array}{l}\text { La chair intervient dans la } \\
\text { fabrication de remèdes contre } \\
\text { le paludisme. }\end{array}$ & \\
\hline Oryctéropes & Chasse au fusil & $\begin{array}{l}\text { La queue intervient dans la } \\
\text { fabrication des anti-poisons, } \\
\text { et dans la confection de } \\
\text { bracelet donnant une grande } \\
\text { force physique. }\end{array}$ & $\begin{array}{l}\text { Toucher le nez ou le bout de la queue pourrait } \\
\text { donner la lèpre. La patte antérieure gauche sert } \\
\text { à confectionner un fétiche capable d'arrêter/ de } \\
\text { faire la pluie ou de donner la mort. }\end{array}$ \\
\hline Phacochères & $\begin{array}{l}\text { Chasse au fusil, avec ou sans } \\
\text { chiens, par piégeage. }\end{array}$ & $\begin{array}{l}\text { La chair est un remède contre } \\
\text { les maux de ventre et le } \\
\text { rhumatisme articulaire. Les } \\
\text { défenses servent à soigner les } \\
\text { lombalgies et à conserver } \\
\text { divers talismans de } \\
\text { protection. }\end{array}$ & $\begin{array}{l}\text { Les défenses servent à confectionner des } \\
\text { bijoux. La crinière sert à fabriquer le chasse } \\
\text { mouche des grands chasseurs. } \\
\text { La queue est utilisée comme trophée; Il est } \\
\text { interdit aux jeunes femmes de voir la queue. } \\
\text { Totem des musulmans. }\end{array}$ \\
\hline Porc-épics & $\begin{array}{l}\text { Chasse au fusil; parfois par } \\
\text { piégeage. }\end{array}$ & $\begin{array}{l}\text { Les piquants et la queue } \\
\text { servent à soigner les maux } \\
\text { d'oreille. }\end{array}$ & $\begin{array}{l}\text { La crinière sert à orner les habits des griots et } \\
\text { des chasseurs, et à décorer les masques de } \\
\text { danse des forgerons. }\end{array}$ \\
\hline Rats & $\begin{array}{l}\text { Piégeage, battues, avec ou sans } \\
\text { chien, par destruction des } \\
\text { tanières. }\end{array}$ & $\begin{array}{l}\text { La peau sert à conserver } \\
\text { divers fétiches. }\end{array}$ & Totem des habitants de Nafoun. \\
\hline
\end{tabular}




\section{DISCUSSION}

La faune de la FCB était jusqu'ici peu connue (Anonyme, 1992). Les rares travaux sur ce sujet ont été effectués par Yaokokoré-Béibro (1995) et Kassé et al. (2006). Des études menées dans le Parc National de la Comoé (PNC, 1150000 ha) à l'Est de la FCB, ont fait état de 60 espèces de mammifères (FGU-KRONBERG, 1979). Dans le Parc National de la Marahoué (PNM, 110000 ha), au Centre de la Côte-d'Ivoire, 48 espèces ont été identifiées (Hoppe-Dominik, 1989). Comparée à ces parcs, la FCB (26 980 ha) qui est 42 fois plus petite que le PNC et 4 fois plus petite que le PNM, avec ses 45 espèces de mammifères, possède une richesse spécifique importante. On trouve dans la FCB de nombreuses espèces également présentes dans le PNC et dans le PNM. Bien que la faune des mammifères de savane prédomine dans la $\mathrm{FCB}$, son territoire recèle également d'une série d'espèces de forêt humide (Kassé et al, 2006), telles que le Céphalophe de Maxwell (Cephalophus maxwellii), le Céphalophe noir (Cephalophus niger), le Céphalophe à dos jaune (Cephalophus silvicultor), Céphalophe à flancs roux (Cephalophus rufilatus), Céphalophe à bande dorsale noire (Cephalophus dorsalis), etc.

En FCB, comme ailleurs dans de nombreux pays, la faune sauvage entre dans l'alimentation des communautés (Adriaens, 1951 ; Child, 1970 ; Ajayi, 1971 ; Asibey, 1974 ; De Vos, 1992 ; Caspary et al. 2001). Comme dans diverses parties du monde, de nombreux facteurs culturels et religieux réglementent la consommation de certaines espèces d'animaux sauvages (De Vos, 1992). Certains villages défendent ainsi à leurs membres de consommer 'un animal qui, selon la tradition, aurait aidé, d'une manière ou d'une autre, les fondateurs du groupe et qui, par conséquent, doit être vénéré. Selon Quin (1959), les singes verts, l'oryctérope et les porcs-épics ne sont consommés que par les hommes et les jeunes garçons chez le peuple Pédi.

Outre cet aspect des rapports entre les riverains de la FCB et la faune, cette dernière est utilisée pour le bien-être de l'homme, à travers divers objets utiles. En effet, la peau, les piquants, la crinière, les cornes, la queue, etc., des animaux sont utilisés pour fabriquer divers produits utiles à l'homme (tam-tams, coras, balafons, sacs, couchettes, tapis de prière, etc.). Dans la FCB, sur les 45 espèces de mammifères répertoriées, en moyenne 15 sont utilisées en médecine traditionnelle. L'usage des animaux sauvages comme remède est pratiqué dans de nombreux autres pays africains. Ainsi, au Ghana, les rats Rattus rattus sont donnés aux enfants atteints de coqueluche (Asibey, 1974).

\section{CONCLUSION}

L'étude a permis de montrer que les mammifères de la FCB interviennent, pour une part importante, dans la vie quotidienne des populations riveraines. Ils représentent une faune très diversifiée. L'étude a montré que la quasitotalité des espèces sont chassés et gagneraient à bénéficier de mesure de conservation contre le braconnage.

Cette faune fait l'objet d'usage divers de la part des riverains. Elle intervient pour une part importante dans l'alimentation, la culture, l'art et la religion, la médecine traditionnelle et le commerce. Au plan alimentaire, de nombreuses espèces sont consommées. Au niveau de la culture et de la religion, certaines espèces sont considérés comme des totems et ne peuvent, par conséquent être abatues, ni être consommées par les populations ou par un groupe social donné. Certains par contre, doivent faire l'objet de rituel avant consommation. D'autres encore sont utilisés pour confectionner des fétiches censés donner des pouvoirs "magiques". La faune entre également dans la fabrication de divers autres produits utiles. En médécine, certains mammifères sont réputés avoir des vertus thérapeutiques et interviendraient dans le traitement de diverses maladies. Enfin au plan commercial, le gibier abattu est vendu occasionnellement par les paysans, alors qu'il fait l'objet d'un trafic quasi-permanent de la part des braconniers. La gestion rationnelle de la faune devrait permettre une autosuffisance en viande de gibier et générer des ressources financières importantes. Ceci serait possible grâce à une chasse réglementée. Enfin cette étude permet, au niveau local, de mieux appréhender les relations étroites entre l'homme et son milieu. 


\section{REFERENCES}

Adriaens E. L. 1951. Recherches sur l'alimentation des populations du Kwango. Bulletin d'Agriculture Congo belge. 42(3) : $473-552$.

Ajayi S. S. 1971. Wildlife as a source of protein in Nigeria : some priorities for development. The Nigerian Field. 36 (3) : 115 - 127.

Anonyme 1. 1993. Biodiversité africaine: fondement pour l'avenir. Un cadre pour intégrer la conservation de la Biodiversité et le développement durable. Programme d'appui à la biodiversité, Washington D. C. $168 \mathrm{p}$.

Anonyme 2. 1992. Aménagement de la forêt classée de Badénou (Rapport), SODEFOR, Abidjan, pp 3 - 15.

Asibey Z. O. A. 1974. Wildlife as a source of protein in Africa north of Sahara. Bio. Conservation. $6: 32$ - 39.

Byers B. A. 1997. Démarche pour comprendre et influencer les comportements à l'égard de la conservation et de la gestion des ressources naturelles. Bulletins sur la biodiversité africaine. $4: 1$ - 133 .

Brousseau, M.F. 1992a. Faune sauvage et faune domestique en milieu pastoral : une synthèse bibliographique. Gibier Faune Sauvage. $9: 167$ - 186.

Brousseau M. F. 1992b. Interaction milieu-faune sauvage au travers des activités pastorales: une synthèse bibliographique. Gibier Faune Sauvage. 9 : 269 - 279.

Caspary H. U., Koné I., Prouot C. et M. De Pauw. 2001. La chasse et la filière viande de brousse dans l'espace Taï, Côte d'Ivoire. Tropenbos. Série 2. 170 p.

Child G. S. 1970. Quand le gibier fournit la viande. Unasylva. $26: 24$ - 29.

Daniel Y. A. 1992. La survie des forêts tropicales. La Recherche. 244 (23) : 692 - 702.

De Vos A. 1992. Le gibier dans l'alimentation : son importance en Afrique et en Amérique du Sud. Unasylva. 29 : 2 - 12.
Gouet J. P., Richard M., Bloc D., Delauge A., Desecures J. P., Dolz J., Maumene J., Paris C., Tranchefort J. et J. Weiss. 1978. L'élaboration d'un protocole d'enquête : proposition d'un plan détaillé et quelques commentaires. Bureau d'études statistiques de l'LT.C.F, Paris. 98 p.

Haldik A. 1992. Pourquoi et comment les forêts tropicales disparaissent. L'agroforesterie ou comment multiplier la biodiversité. La Recherche. 244 (23) : 698 - 699.

Haltenorth Th., Diller H. et M. Cuisin. 1985. Mammifères d'Afrique et de Madagascar. Delachaux et Niestlé, Paris. 397 p.

Hoppe-Dominik B. 1989. Premier recensement des grands Mammifères dans le Parc National de la Marahoué, en Côte d'Ivoire. Rev. Zoo.Afr., 103 : 21 - 27.

Kassé K. B., Kadjo B., Yaokokoré-Béibro K. H. et K. Foua-Bi. 2006. Inventaire, distribution et mesure de conservation des grands mammifères de la forêt classée de Badénou (Nord de la Côte d'Ivoire) Rev. Ivoir. Sci. Technol., 07 : 173 - 188

FGU-KRONBERG 1979. Etat actuel des parcs nationaux de la Comoé et de Taï ainsi que la réserve d'Azagny et propositions visant à leur conservation et à leur développement aux fins de promotion du tourisme. Tome 2, partie 1 : Parc National de la Comoé. Inventaire des conditions écologiques et biologiques. $140 \mathrm{p}$.

Quin P. J. 1959. Food and feeding habits of the Pedi, Johannesburg, Witwatersrand University Press, $278 \mathrm{p}$.

Wolf E. W. 1991. Restauration d'un écosystème forestier : contribution de l'aménagement de la faune. Séminaire sur l'aménagement intégré des forêts denses humides et des zones agricoles périphériques. Abidjan, $12 \mathrm{p}$.

Yaokokoré-Béibro K. H. 1995. Contribution à l'étude ethnozoologique de la forêt classée de Badénou (Korhogo) : cas des Mammifères. Mémoire de D.E.A. d'Ecologie Tropicale, Université d'Abidjan, $55 \mathrm{p.}$ 